\title{
Illness perceptions and stress: mediators between disease severity and psychological well-being and quality of life among patients with Crohn's disease
}

This article was published in the following Dove Press journal:

Patient Preference and Adherence

23 November 2016

Number of times this article has been viewed

\author{
Maochen Zhangl,* \\ Liwen Hong',* \\ Tianyu Zhang' \\ Yun Lin' \\ Sichang Zheng' \\ Xiaolin Zhou' \\ Rong Fan' \\ Zhengting Wang' \\ Chenli Zhang ${ }^{2}$ \\ Jie Zhong' \\ 'Department of Gastroenterology, \\ ${ }^{2}$ Exclusive Medical Center, Ruijin \\ Hospital, School of Medicine, Shanghai \\ Jiao Tong University, Shanghai, People's \\ Republic of China \\ *These authors contributed equally \\ to this work
}

Background: Disease severity, illness perceptions, coping strategies, stress, psychological well-being, and quality of life were reported to have close relationships. According to the Common Sense Model, illness perceptions and coping strategies could mediate the relationship between illness stimuli and illness outcomes such as psychological health and quality of life. Stress was also associated with the individual's disease severity, anxiety, depression, and quality of life.

Objectives: The study aimed to explore the influencing factors of illness outcomes, and to what extent illness perceptions, coping strategies, and stress mediate the relationship between disease severity and anxiety, and depression and quality of life.

Methods: Our study included 159 patients with Crohn's disease who were attending a tertiary hospital outpatient clinic or who were hospitalized. Disease severity was measured with the Crohn's Disease Activity Index. Illness perceptions were measured with the Brief Illness Perceptions Questionnaire. Coping strategies were measured with the Carver Brief Coping Questionnaire. Stress was measured with the Perceived Stress Questionnaire. Anxiety and depression were measured with the Hospital Anxiety and Depression Scale. Quality of life was measured with the Inflammatory Bowel Disease Questionnaire.

Results: Disease severity, illness perceptions, maladaptive coping, stress, anxiety, depression and quality of life were significantly correlated with each other among patients with Crohn's disease. Using structural equation modeling to describe the inner relationship of the aforementioned variables, an excellent-fitted model was drawn. $\left(\chi^{2}[10]=13.83, P=0.18, \chi^{2} / \mathrm{N}=1.38\right.$, standardized root mean square residual [SRMR] $<0.05$, root mean square error of approximation $[\mathrm{RMSEA}]<0.05$, goodness of fit index [GFI] $>0.97$, comparative fit index $[\mathrm{CFI}]>0.99$ ). Disease severity had a direct influence on illness perceptions. Illness perceptions had a direct influence on stress. Both illness perceptions and stress had direct influences on anxiety, depression, and quality of life, while maladaptive coping did not directly influence anxiety, depression, or quality of life. Stress had a direct influence on maladaptive coping. Quality of life was also directly influenced by disease severity and anxiety.

Conclusion: Interrelationships between disease stimuli, disease perceptions and management and disease outcomes could be found in patients with Crohn's disease. Illness perceptions and stress mediated an individual's disease severity and anxiety, depression and quality of life, while coping strategy was not an applicable mediator.

Keywords: Crohn's disease, disease severity, illness perceptions, coping strategies, stress, psychological well-being, quality of life

\section{Introduction}

Inflammatory bowel disease (IBD), including two major gastrointestinal disorders namely Crohn's disease (CD) and ulcerative colitis, affects nearly 28 million people 
worldwide, ${ }^{1,2}$ and there is an upward tendency in morbidity of the disease. Long time of sickness and repeated flares greatly affect patient's study, work and life, which finally leads to a negative effect on their psychological well-being. Prevalence of psychological distress such as anxiety and depression is common in IBD patients. The rates of anxiety and depression are estimated to be $58 \%$ and $19 \%$ in IBD patients, ${ }^{3}$ which is much higher than the controls $\mathrm{s}^{3-12}$ and other chronic diseases such as diabetes ${ }^{13}$ and rheumatoid arthritis. ${ }^{14}$ Even when compared to individuals with newly discovered cancer of the colon, newly diagnosed patients with IBD seem to have higher levels of anxiety and depression. ${ }^{15}$

Much research has been dedicated to exploring the risk factors associated with the development of anxiety and depression in IBD patients. These include disease activity, ${ }^{3,16-25}$ surgery and stoma, ${ }^{26,27}$ psychological stress ${ }^{28,29}$ and health quality of life. ${ }^{16,17,23,26,29}$ To have a better understanding of the inner relationship between these factors and psychological health is important, so that clinical interventions can be made to improve patient's psychological status.

Patient's health behaviors, psychological well-being and adherence to medication can be determined by their perception and cognition of the disease. Social cognitive models provide theoretical evidence for the influences of perceptional and cognitive factors to patient's self-management. One of the validated models focusing on the relationship between cognitive factors and illness outcomes is the Common Sense Model (CSM) of illness representations. ${ }^{30}$ According to the CSM, patients generate cognitive representations based on the information regarding their disease, and then these beliefs can impinge on their coping strategies and illness outcomes. Appraisal and evaluation are made by patients due to the success of the illness outcomes, which feeds back information to each previous stage and leads to changes in their illness representations and coping strategies. Therefore, illness perceptions and coping strategies are posited to be two important mediators between individual's illness status and illness outcomes such as psychological well-being and quality of life.

Illness perceptions are composed of five dimensions, ${ }^{31}$ including cause, consequences, identity, timeline and cure/ controllability. The cause dimension represents the cognition about the potential causes responsible for the disease. Consequences refer to the impact of the illness on an individual's life. Identity represents the beliefs about the illness label and knowledge about its symptoms. Timeline means individual's beliefs about how long the disease will last. Cure or controllability refers to the belief about the efficacy of treatment.

Coping may be defined as an individual's efforts to deal with the difficult situations they are faced with. ${ }^{32} \mathrm{~A}$ wide range of classification is available for coping styles and the coping styles that are more commonly used are problemfocused coping versus emotion-focused coping and adaptive coping versus maladaptive coping. Problem-focused coping aims to distract the source of tough situations while emotionfocused coping aims to improve emotional moods. Adaptive coping can reduce distress whereas maladaptive coping can increase distress.

Studies for the effectiveness of the CSM have already been made in a variety of diseases. For instance, patients with chronic fatigue syndrome who considered their disease as serious and not controllable and who coped their illness in a negative way seemed to have higher levels of impairment in physical health and reported greater problems about psychological well-being and social functioning. ${ }^{33}$ Similar results are identified in other chronic diseases such as psoriasis, ${ }^{34,35}$ diabetes, ${ }^{36,37}$ Addison's disease, ${ }^{38}$ asthma, ${ }^{39}$ rheumatoid arthritis, ${ }^{35,39}$ breast cancer ${ }^{40}$ and COPD. ${ }^{35}$

Dorrian et $\mathrm{al}^{41}$ applied the CSM in an IBD cohort for the first time. In their research, illness perceptions predicted adjustment while coping did not mediate the relationship between illness perceptions and adjustment as the CSM proposed. However, in subsequent studies, Knowles et al ${ }^{42,43}$ found there was an interrelationship between disease activity, illness perceptions, coping strategies and anxiety and depression among IBD patients and those with stomas. They also constructed structural equation modeling (SEM) to describe the correlations between the CSM variables. ${ }^{42,43}$

Stress, which refers to the state of mental or emotional tension, also troubled IBD patients. Chronic diseases can cause high levels of stress and in turn stress can influence the development of chronic diseases. Keegan et $\mathrm{al}^{29}$ found that individual's stress was associated with disease severity, quality of life and psychological health such as anxiety and depression. So, stress could be a probable mediator between IBD patient's illness status and illness outcomes and could play an important role in the progress of IBD.

Till date, very few research about the application of the CSM in IBD patients have been made among Asian people. Besides, to what extent stress can influence an individual's physical and psychological health is also known little. The aim of our study was to examine the utility of the CSM variables and stress in Chinese patients diagnosed with $C D$ and predict the mediators between illness status and illness outcomes of IBD patients. We hypothesized there would be an interrelationship between disease severity, illness perceptions, maladaptive coping, stress, anxiety, depression and quality of life. We also predicted illness perceptions, coping strategies and stress could mediate the relationship 
between disease severity and illness outcomes such as anxiety, depression and quality of life.

\section{Materials and methods Participants}

This study was approved by the ethics committee of Ruijin Hospital affiliated to School of Medicine Shanghai Jiao Tong University, and all the patients provided written informed consent. A total of 159 patients with $\mathrm{CD}$ attending an outpatient clinic or hospitalized in the Department of Gastroenterology, Ruijin Hospital, School of Medicine, Shanghai Jiao Tong University from July 1, 2015 to December 31, 2015, were selected for the study.

Inclusion criteria were as follows: definite diagnosis of $\mathrm{CD}$, no history of mental disease, no history of psychological intervention history, absence of other chronic diseases and ability to understand and finish the questionnaires independently and voluntarily.

\section{Assessment and questionnaires Disease severity}

The Crohn's Disease Activity Index (CDAI) ${ }^{44}$ is a 9-item questionnaire to measure the clinical disease severity of patients with $\mathrm{CD}$. The contents include stool frequency, abdominal pain, general well-being, extraintestinal symptoms such as fistula and fever, use of antidiarrheal agents, abdominal mass, hematocrit, height and weight. Higher scores indicate higher clinical disease severity: less than 150 (inactive) and greater than 150 (active).

\section{Illness perceptions}

The Brief Illness Perceptions Questionnaire (BIPQ $)^{45}$ is a validated 9-item questionnaire to assess an individual's perceptions and cognitions on their disease. The questions measure 8 dimensions: consequences, timeline, personal control, treatment control, identity, concern, understanding and emotional response. Each item is scored with a 11-point scale. For example, "How much does your illness affect your life?": from 0 (did not affect at all) to 10 (severely affects my life). After specific items are reverse-scored, higher total scores indicate poorer illness cognition.

\section{Coping strategies}

The Brief Coping Operations Preference Enquiry (Brief COPE) ${ }^{46}$ is a validated 28 -item instrument to assess coping behaviors known to be of adaptive and maladaptive strategies. Each item is measured with a 4-point scale ranging from 0 (not at all) to 3 (a lot or all of the time). The coping strategies are divided into adaptive problem-focused coping (active coping, planning and using instrumental support), adaptive emotion-focused coping (positive reframing, acceptance, humor, religion and using emotional support) and maladaptive coping (self-distraction, denial, venting, substance use, behavioral disengagement and self-blame)..$^{32}$ Higher scores in each group indicate more use of the particular coping strategy.

\section{Stress}

The Perceived Stress Questionnaire (PSQ) ${ }^{47}$ is a validated 30-item measure to evaluate a patient's level of perceived stress. The contents include 7 dimensions: harassment (4 items), overload (4 items), irritability ( 2 items), lack of joy ( 7 items), fatigue ( 4 items), worries ( 5 items) and tension (4 items). Each item is measured with a 4-point scale from 1 (almost never) to 4 (usually). After several items are reversescored, higher scores indicate greater perceived stress.

\section{Anxiety and depression}

The Hospital Anxiety and Depression Scale (HADS) ${ }^{48}$ is a 14-item questionnaire ( 7 items for anxiety and 7 items for depression) to measure an individual's level of anxiety and depression. Each item is scored with a 4-point scale. For example, "I feel tense or "wound up"': from 0 (not at all) to 3 (most of the time). Total scores of each subscale range from 0 to 21. Higher scores represent higher levels of anxiety or depression: 0-7 (normal), 8-10 (mild), 11-15 (moderate) and 16-21 (severe).

\section{Quality of Life}

The Inflammatory Bowel Disease Questionnaire (IBDQ) $)^{49}$ is a validated 32-item instrument to measure the health quality of life particularly for IBD patients. The questions evaluate 4 dimensions: bowel symptoms (10 items), systemic symptoms (5 items), emotional function (12 items) and social function (5 items). Each item is scored with a 7-point scale, from 1 (worst) to 7 (best). Total scores range from 32 to 224 with lower scores indicating poorer quality of life.

\section{Data collection}

Participants were told the objective and method of the research in advance. With their permission, the participants were informed of the requirements of the questionnaires; they completed them independently within 20-30 minutes. All the questionnaires were taken back immediately.

\section{Statistical analysis}

Pearson product moment correlation analysis was used to compare the relationship between disease severity (CDAI), 
illness perceptions (BIPQ), coping strategies (Brief COPE), stress (PSQ), anxiety and depression (HADS) and quality of life (IBDQ). The SEM based on the CSM variables and stress was drawn using the Amos statistical package $\left(\chi^{2} P>0.05\right.$, $\chi^{2} / \mathrm{N}=1-3$, SRMR $<0.05$, RMSEA $<0.05$, GFI $>0.9$, $\mathrm{CFI}>0.9$ indicated excellent model fit). The nonsignificant pathways ( $P$-values of standardized path coefficients $>0.05$ ) or variables (problem-focused coping and emotion-focused coping) were removed in the final model. Each dimension of the illness perceptions (BIPQ), coping strategies (Brief COPE) and stress (PSQ) was picked out individually and was computed to anxiety, depression and quality of life using correlational analysis.

\section{Results}

The proportion of different levels of anxiety and depression of the 159 participants is shown in Table 1. It can be seen that $57.23 \%$ individuals had mild anxiety and $25.79 \%$ had moderate to severe anxiety. Also $44.03 \%$ patients had mild depression and $15.72 \%$ had moderate to severe depression.

As shown in Table 2, correlations exist between disease severity, illness perceptions, maladaptive coping, stress, anxiety, depression and quality of life. Disease severity and illness perceptions had significant positive correlations with maladaptive coping, stress, anxiety and depression. Besides, disease severity and illness perceptions had significant negative correlations with quality of life. This indicated that patients with more serious disease status and poorer illness perceptions used more maladaptive coping strategies and had more perceived stress, higher levels of anxiety and depression and lower quality of life. Disease severity and illness perceptions also significantly correlated with each other indicating that patients suffering more symptoms thought their disease more hopeless. However, no significant correlations between disease severity, illness perceptions and problem-focused coping or emotion-focused coping were found.

Maladaptive coping had a positive correlation with stress, anxiety and depression, and it also had a negative correlation with quality of life, suggesting the utility of maladaptive coping strategies was associated with psychological stress, psychological well-being and quality of life. No such associations were found for problem-focused coping and

Table I Proportion of different levels of anxiety and depression

\begin{tabular}{lllll}
\hline & $\begin{array}{l}\text { Normal } \\
(\mathbf{0 - 7 )}\end{array}$ & $\begin{array}{l}\text { Mild } \\
\mathbf{( 8 - 1 0 )}\end{array}$ & $\begin{array}{l}\text { Moderate } \\
(\mathbf{I I - 1 5 )}\end{array}$ & $\begin{array}{l}\text { Severe } \\
(\mathbf{I 6 - 2} \mathbf{)})\end{array}$ \\
\hline Anxiety (\%) & 16.98 & 57.23 & 25.16 & 0.63 \\
Depression (\%) & 40.25 & 44.03 & 15.72 & 0 \\
\hline
\end{tabular}

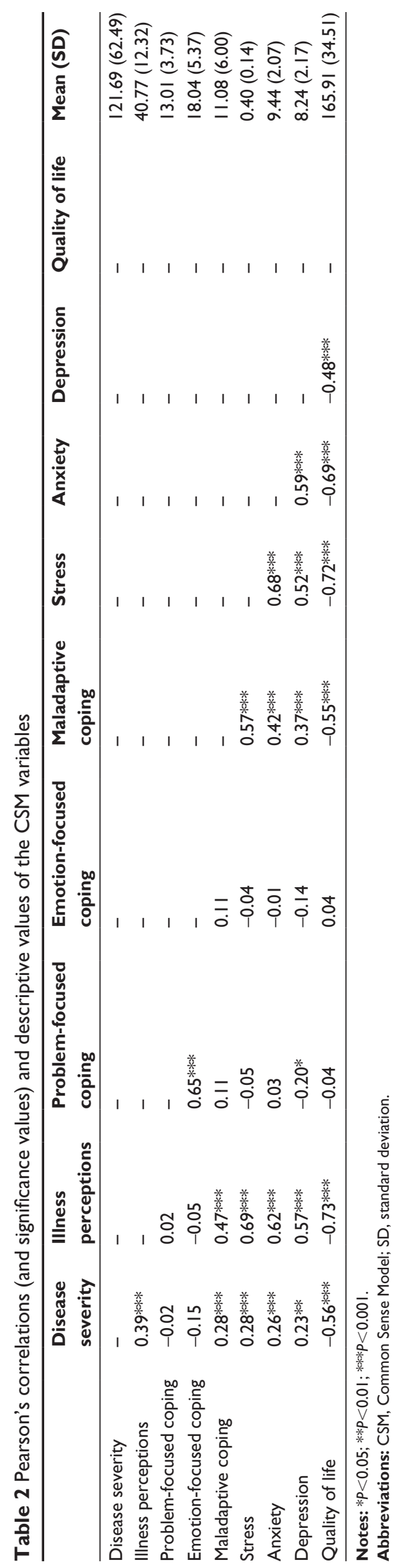


emotion-focused coping. Within the three different styles of coping strategies, problem-focused coping correlated with emotion-focused coping indicating that individuals were likely to choose problem-focused coping and emotionfocused coping at the same time. However, maladaptive coping had no relations with problem-focused coping or emotion-focused coping.

Stress was found to be positively correlated with anxiety and depression and negatively correlated with quality of life suggesting that there was an association between higher level of stress and poorer psychological health and quality of life. Anxiety and depression also correlated with each other showing that psychologically distressed individuals were likely to be in the status of both anxiety and depression. Both anxiety and depression had significant negative correlations with quality of life suggesting that psychological status had association with physiological well-being.

The association between different dimensions of illness perceptions, coping strategies, stress and psychological wellbeing and quality of life is shown in Tables $3-5$. The results show that consequences, timeline, personal control, treatment control, identity, concern and emotional response are significantly correlated with anxiety, depression and quality of life. These results indicate that patients who thought their disease serious, chronic, uncontrollable and disturbing were more likely to have psychological distress and poor quality of life. However, understanding of the disease was not correlated with anxiety, depression or quality of life.

Planning and positive reframing had negative correlations with depression suggesting that making plans and seeing things in a positive way were associated with less depression. It can be seen that humor negatively correlated with depression and positively correlated with quality of life, while religion positively correlated with anxiety and negatively correlated with quality of life, which indicated humor was beneficial to psychological well-being while

Table 3 Pearson's correlations (and significance values) between illness perceptions, psychological measures and quality of life

\begin{tabular}{llll}
\hline & Anxiety & Depression & Quality of life \\
\hline Consequences & $0.52^{* * *}$ & $0.43^{* * *}$ & $-0.56^{* * *}$ \\
Timeline & $0.20^{*}$ & $0.31^{* * *}$ & $-0.22^{* * *}$ \\
Personal control & $0.31^{* * *}$ & $0.20^{*}$ & $-0.43^{* * *}$ \\
Treatment control & $0.18^{*}$ & $0.16^{*}$ & $-0.30^{* * *}$ \\
Identity & $0.31^{* * *}$ & $0.31^{* * *}$ & $-0.38^{* * *}$ \\
Concern & $0.57^{* * *}$ & $0.48^{* * *}$ & $-0.62^{* * *}$ \\
Understanding & 0.07 & 0.09 & -0.09 \\
Emotional response & $0.57^{* * *}$ & $0.51^{* * *}$ & $-0.63^{* * *}$
\end{tabular}

Notes: $* P<0.05 ; * * * P<0.001$.
Table 4 Pearson's correlations (and significance values) between coping strategies, psychological measures and quality of life

\begin{tabular}{llll}
\hline & Anxiety & Depression & Quality of life \\
\hline Active coping & 0.01 & $-0.20^{*}$ & -0.01 \\
Planning & -0.08 & $-0.31^{* * *}$ & 0.08 \\
Positive $\mathrm{r}$ & $-0.18^{*}$ & $-0.25^{* * *}$ & $0.20^{*}$ \\
Acceptance & -0.13 & $-0.19^{*}$ & 0.15 \\
Humor & -0.15 & $-0.22^{* *}$ & $0.22^{* *}$ \\
Religion & $0.31^{* * *}$ & $0.17^{*}$ & $-0.22^{* *}$ \\
Using emotional support & 0.05 & -0.02 & -0.15 \\
Using instrumental support & 0.12 & 0.02 & $-0.16^{*}$ \\
Self-distraction & 0.15 & 0.02 & $-0.19^{*}$ \\
Denial & $0.32^{* * *}$ & $0.19^{*}$ & $-0.33^{* * *}$ \\
Venting & $0.43^{* * *}$ & $0.42^{* * *}$ & $-0.58^{* * *}$ \\
Substance use & 0.16 & $0.16^{*}$ & $-0.27^{* * *}$ \\
Behavioral disengagement & $0.28^{* * *}$ & $0.17^{*}$ & $-0.35^{* * *}$ \\
Self-blame & $0.19^{*}$ & $0.32^{* * *}$ & $-0.25^{* *}$ \\
\hline
\end{tabular}

Notes: $* P<0.05 ; * * P<0.01 ; * * * P<0.001$.

religion had an unfavorable effect. Also denial and behavioral disengagement correlated with anxiety and quality of life, while self-blame correlated with depression and quality of life. Association between venting and anxiety, depression and quality of life was also discovered. Substance use had a negative correlation with quality of life. These results showed that engagement of denial, venting, substance use, behavioral disengagement and self-blame to some extent was associated with higher levels of anxiety, depression and lower quality of life.

It can be seen that all the 7 dimensions in PSQ were positively correlated with anxiety and depression. They also negatively correlated with the quality of life. This suggests that stress (regardless of the type or form) was strongly associated with psychological status and quality of life.

All the studied variables including disease severity, illness perceptions, coping strategies, stress, anxiety, depression and quality of life were considered for SEM. Using modification indices, all the nonsignificant pathways were removed. The final SEM model is shown in Figure 1 and has an excellent

Table 5 Pearson's correlations (and significance values) between stress, psychological measures and quality of life

\begin{tabular}{llll}
\hline & Anxiety & Depression & Quality of life \\
\hline Harassment & $0.52 * * *$ & $0.39 * * *$ & $-0.53 * * *$ \\
Overload & $0.26 * * *$ & $0.20 *$ & $-0.30 * * *$ \\
Irritability & $0.49 * * *$ & $0.51 * * *$ & $-0.52^{* * *}$ \\
Lack of joy & $0.62 * * *$ & $0.57 * * *$ & $-0.55^{* * *}$ \\
Fatigue & $0.61 * * *$ & $0.53 * * *$ & $-0.72 * * *$ \\
Worries & $0.66 * * *$ & $0.53 * * *$ & $-0.71 * * *$ \\
Tension & $0.60 * * *$ & $0.52 * * *$ & $-0.65 * * *$ \\
\hline
\end{tabular}

Notes: $* P<0.05 ; * * * P<0.001$. 


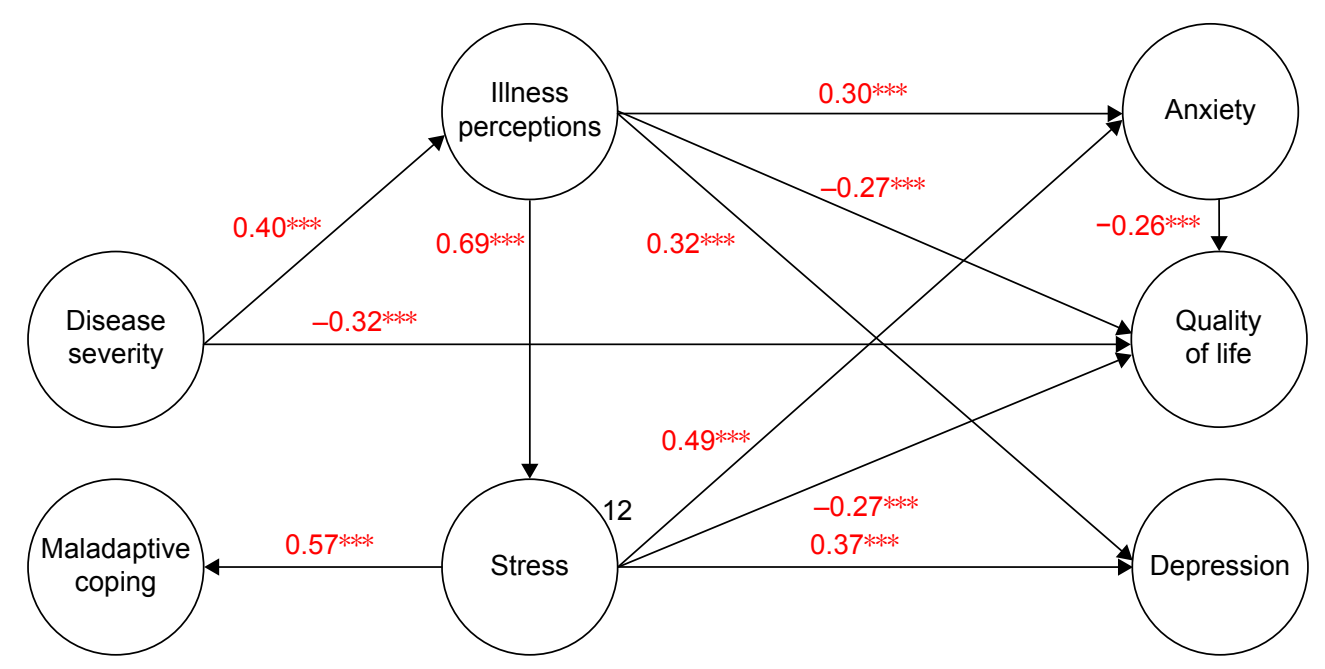

Figure I Final CSM (nonsignificant pathways or variables removed, $* * * P<0.001$ ).

Notes: The values represent standardized path coefficients.

Abbreviation: CSM, Common Sense Model.

fit $\left(\chi^{2}[10]=13.83, P=0.18, \chi^{2} / \mathrm{N}=1.38\right.$, standardized root mean square residual [SRMR] $<0.05$, root mean square error of approximation [RMSEA] $<0.05$, goodness of fit index $[\mathrm{GFI}]>0.97$, comparative fit index $[\mathrm{CFI}]>0.99$ ). Disease severity had a significant direct influence on illness perceptions. Illness perceptions had significant influences on stress, anxiety, depression and quality. Stress had a significant influence on maladaptive coping, anxiety, depression and quality of life. Quality of life was also directly influenced by disease severity and anxiety. Except these direct relations, illness perception mediated the relationship between disease severity and stress, while stress mediated the relationship between illness perceptions and anxiety, depression and quality of life. However, maladaptive coping was not a good mediator between illness perceptions and anxiety, depression and quality of life as the CSM proposed.

\section{Discussion}

The purpose of the current study was to explore the relationships between disease severity, illness perceptions, coping strategies, stress, anxiety, depression and quality of life. Our study also aimed to predict whether illness perceptions, coping strategies and stress were potential mediators between disease severity and psychological well-being and quality of life.

In our cohort, high prevalence of psychological distress can be found, which were similar to previous studies. ${ }^{3-12,41-43}$ Anxiety other than depression seemed to be a more common type of psychological distress in IBD patients.

Disease severity, illness perceptions, maladaptive coping, stress, anxiety, depression and quality of life had intricate and complex relations with each other. Individuals who experienced stronger disease severity seemed to generate poorer illness perceptions, take maladaptive coping strategies more often, suffer higher extent of stress and pressure, bothered with higher levels of psychological problems such as anxiety and depression and undergo lower quality of life; these findings are consistent with previous studies. ${ }^{42,43}$ Among these complicated correlations, some were direct while others were indirect.

Disease severity could directly influence a patient's illness perceptions. Individuals enduring more symptoms might generate more pessimistic cognitions of their disease and think their conditions more hopeless. Such results are consistent with previous research, which proved the relationship between higher disease activity and poorer illness perceptions. ${ }^{41-43}$ Disease severity also had a direct influence on an individual's quality of life. The severity of the flare and the frequency of the relapse intensely affected IBD patient's life well-being. ${ }^{50,51}$

Illness perceptions had direct influences on patient's stress suggesting that poorer illness perceptions could raise mental and emotional tension. Illness perceptions also had direct influences on IBD patient's anxiety, depression and quality of life. Individual's thoughts of their disease being chronic, not controllable and having horrible consequences easily led to high levels of psychological distress and reduction in quality of life. Once they generated negative perceptions and cognitions, the unpleasant feelings finally converted into negative psychological well-being. Similar results were also found in other research. ${ }^{28,41-43,52}$ However, inconsistent with the CSM and Knowles et al's studies, ${ }^{42,43}$ our research 
showed illness perceptions did not have any direct influence on patient's coping strategies. But our study found the direct influence of illness perceptions on stress and the direct influence of stress on maladaptive coping, indicating that stress mediated the relationship between illness perceptions and maladaptive coping.

Maladaptive coping did not directly influence anxiety, depression or quality of life, contrary to the CSM and the study made by Knowles et al..$^{43}$ The probable reason responsible for obtaining different results was that the Brief COPE was a questionnaire designed for a variety of diseases, not for IBD patients specifically. ${ }^{41,53}$ Many of the coping strategies evaluated in this questionnaire could not accurately reflect IBD patient's real conditions. Recently, McCombie et al ${ }^{53}$ developed a concise IBD-specific coping strategy questionnaire named IBD-COPE. Although whether the new scale could better represent IBD patient's coping choices needed further exploration, it could be put into use in our future studies to measure the role of coping strategies played in the course of IBD. Nevertheless, consistent with previous studies, ${ }^{28,32,42,43,52}$ the correlations between coping strategies especially maladaptive coping (denial, venting, behavioral engagement and self-blame) and psychological well-being and quality of life did exist, emphasizing that some factors (among them might include illness perceptions, disease severity, and stress) connected coping strategies and psychological well-being and quality of life in an indirect way. Regarding emotion-focused coping and problem-focused coping, such correlations were not found.

Stress had direct influences on anxiety, depression and quality of life of IBD patients. This indicates that perceived stress, regardless of its types and forms, including harassment, overload, irritability, lack of joy, fatigue, worries and tension, could lead to mood disorders and undesirable living situations. Consistent with the previous studies, this study found a higher level of perceived stress to be an important determinant and strong predictor of anxiety, depression and quality of life. ${ }^{28,29,54,55}$ Anxiety had a direct influence on quality of life while depression did not have such functioning, proposing that among the different types of psychological distress, anxiety pretended to have most serious effects on an individual's life. This result was in accordance with our findings that the prevalence of anxiety was higher than depression.

Based on the CSM theory, patients will generate different illness representations for the same disease according to their own symptomatic and emotional features. Further, they make characteristic coping plans and consequently get different illness outcomes. ${ }^{30}$ Thus illness stimuli can affect psychological outcomes and quality of life indirectly by means of illness perceptions and coping. In our study, illness perceptions could mediate the relationship between disease severity and anxiety, depression and quality of life, which is in accordance with the CSM theory and with the results by Knowles et al..$^{42,43}$ However, none of the emotion-focused coping, problem-focused coping or maladaptive coping could mediate the relation between illness perceptions and illness outcomes. This result was contrary to the CSM and previous research. ${ }^{42,43}$ The controversy whether coping strategies can mediate illness perceptions and illness outcomes need to be proved by further research. We also found that stress mediated the relationship between illness perceptions and anxiety, depression and quality of life. Therefore, we suppose stress plays a critical role in mediating disease severity and illness outcomes.

Given the epidemiological data of increased risk of psychological disorders and difficulties among IBD patients and growing evidence for the relation between psychological disorders and disease activity, the engagement of psychological interventions in IBD management seemed required. ${ }^{56,57}$ Selective serotonin reuptake inhibitors and serotonin norepinephrine reuptake inhibitors such as duloxetine were effective pharmacologic treatments for psychological distress among IBD patients. ${ }^{58,59}$ Compared with standard-care treated patients, those who received additional cognitive behavioral therapy reported greater improvements in anxiety, depression and quality of life. ${ }^{60-62}$ All the aforementioned therapies are aimed at anxiety and depression directly. Since our study found illness perceptions and stress were mediators between disease status and psychological status, interventions targeting these two factors would also be probably effective in improving individual's anxiety, depression and quality of life. We should lay more emphasis on patient's health education and pressure relief. For example, distribution of a brief and integrated brochure introducing the disease to every IBD patient might be a simple but effective way to improve patient's illness perceptions, which was finally beneficial to illness outcomes. ${ }^{63}$ Any interventions which can relieve stress such as music, enough sleep, moderate exercise, communication with friends and massage might bring about improvements in psychological well-being and quality of life as well. ${ }^{64,65}$ What is more, these methods are quite easy to take into practice and have almost no side effects.

\section{Limitations}

There were many limitations in our study. First, samples of more than 200 were recommended in research of SEM. 
Although we got an excellent-fitted model, studies of larger samples were needed to prove our findings. Second, in our study CDAI, which was used to evaluate individual's disease severity, was a scale based on patient's symptoms, only representing their clinical disease activity without measuring mucosal inflammation. Some research showed poor correlation between CDAI and biological markers of inflammation (such as hsCRP, fecal calprotectin and lactoferrin) and endoscopic disease activity (such as Crohn's Disease Endoscopic Index of Severity [CDEIS] and Simple Endoscopic Score for Crohn's Disease [SES-CD]), suggesting clinical disease severity could not best reflect mucosal inflammation. ${ }^{66-68}$ Therefore, in future studies, biological markers and endoscopic evaluation used together with CDAI would better represent individual's disease severity. Third, most of the variables and questionnaires in our research had already been studied in previous research. ${ }^{42,43}$ However, stress and quality of life were two new variables added in our study which were not included in previous studies. Besides, we got different results about the role that coping strategies played in the SEM. Finally, most of the previous studies and our research were cross-sectional studies. The CSM mentioned a feedback in each step indicating the variables we studied would change from time to time in the course of disease. In our future studies, follow-up evaluation should be conducted and patients with different kinds of medication should be studied separately and compared with each other so that we could tell out the advantages and disadvantages of each treatment regarding illness perceptions, coping strategies, stress, anxiety, depression and quality of life of in IBD patients.

\section{Conclusion}

Our study proved the inner relationships between more severe disease status, poorer illness perceptions, more maladaptive coping strategies use, greater perceived stress, higher levels of anxiety, depression and lower quality of life in IBD patients. Illness perceptions and stress mediated individual's disease severity and anxiety, depression and quality of life, while coping strategies did not have such functioning. Interventions aimed at improving illness perceptions and reducing perceived stress could be probably effective in improving the psychological health and quality of life of IBD patients.

\section{Acknowledgment}

The authors would like to thank Shurong Hu and Mengmeng Cheng for their help in the study. This study was supported by National Natural Science Foundation of China (nos 81670503 and 81602558) and Shanghai Committee of Science and Technology Foundation (no 16411950408).

\section{Author contributions}

All authors contributed toward data analysis, drafting and revising the paper and agree to be accountable for all aspects of the work.

\section{Disclosure}

The authors report no conflicts of interest in this work.

\section{References}

1. Ananthakrishnan AN. Epidemiology and risk factors for IBD. Nat Rev Gastroenterol Hepatol. 2015;12(4):205-217.

2. Schoultz M, Atherton I, Hubbard G, Watson AJ. Assessment of causal link between psychological factors and symptom exacerbation in inflammatory bowel disease: a protocol for systematic review of prospective cohort studies. Syst Rev. 2013;2:8.

3. Goodhand JR, Wahed M, Mawdsley JE, Farmer AD, Aziz Q, Rampton DS. Mood disorders in inflammatory bowel disease: relation to diagnosis, disease activity, perceived stress, and other factors. Inflamm Bowel Dis. 2012;18(12):2301-2309.

4. Kurina LM, Goldacre MJ, Yeates D, Gill LE. Depression and anxiety in people with inflammatory bowel disease. $J$ Epidemiol Community Health. 2001;55(10):716-720.

5. Addolorato G, Capristo E, Stefanini GF, Gasbarrini G. Inflammatory bowel disease: a study of the association between anxiety and depression, physical morbidity, and nutritional status. Scand J Gastroenterol. 1997;32(10):1013-1021.

6. Tarter RE, Switala J, Carra J, Edwards KL, Van Thiel DH. Inflammatory bowel disease: psychiatric status of patients before and after disease onset. Int J Psychiatry Med. 1987;17(2):173-181.

7. Walker JR, Ediger JP, Graff LA, et al. The Manitoba IBD cohort study: a population-based study of the prevalence of lifetime and 12-month anxiety and mood disorders. Am J Gastroenterol. 2008;103(8): 1989-1997.

8. Berrill JW, Gallacher J, Hood K, et al. An observational study of cognitive function in patients with irritable bowel syndrome and inflammatory bowel disease. Neurogastroenterol Motil. 2013;25(11):918-e704.

9. Lerebours E, Gower-Rousseau C, Merle V, et al. Stressful life events as a risk factor for inflammatory bowel disease onset: a population-based case-control study. Am J Gastroenterol. 2007;102(1):122-131.

10. Loftus EV Jr, Guérin A, Yu AP, et al. Increased risks of developing anxiety and depression in young patients with Crohn's disease. Am J Gastroenterol. 2011;106(9):1670-1677.

11. Piche T, Ducrotté P, Sabate JM, et al. Impact of functional bowel symptoms on quality of life and fatigue in quiescent Crohn disease and irritable bowel syndrome. Neurogastroenterol Motil. 2010;22(6):626-e174.

12. van Langenberg DR, Gibson PR. Factors associated with physical and cognitive fatigue in patients with Crohn's disease: a cross-sectional and longitudinal study. Inflamm Bowel Dis. 2014;20(1):115-125.

13. Engström I. Mental health and psychological functioning in children and adolescents with inflammatory bowel disease: a comparison with children having other chronic illnesses and with healthy children. J Child Psychol Psychiatry. 1992;33(3):563-582.

14. Miehsler W, Weichselberger M, Offerlbauer-Ernst A, et al. Which patients with IBD need psychological interventions? A controlled study. Inflamm Bowel Dis. 2008;14(9):1273-1280.

15. Filipović BR, Filipović BF, Kerkez M, Milinić N, Randelović T. Depression and anxiety levels in therapy-naive patients with inflammatory bowel disease and cancer of the colon. World J Gastroenterol. 2007;13(3): 438-443. 
16. Graff LA, Walker JR, Lix L, et al. The relationship of inflammatory bowel disease type and activity to psychological functioning and quality of life. Clin Gastroenterol Hepatol. 2006;4(12):1491-1501.

17. Calvet X, Gallardo O, Coronas R, et al. Remission on thiopurinic immunomodulators normalizes quality of life and psychological status in patients with Crohn's disease. Inflamm Bowel Dis. 2006;12(8):692-696.

18. Addolorato G, Mirijello A, D'Angelo C, et al. State and trait anxiety and depression in patients affected by gastrointestinal diseases: psychometric evaluation of 1641 patients referred to an internal medicine outpatient setting. Int J Clin Pract. 2008;62(7):1063-1069.

19. Bennebroek Evertsz' F, Thijssens NA, Stokkers PC, et al. Do inflammatory bowel disease patients with anxiety and depressive symptoms receive the care they need? J Crohns Colitis. 2012;6(1):68-76.

20. Cámara RJ, Schoepfer AM, Pittet V, Begré S, von Känel R; Swiss Inflammatory Bowel Disease Cohort Study (SIBDCS) Group. Mood and nonmood components of perceived stress and exacerbation of Crohn's disease. Inflamm Bowel Dis. 2011;17(11):2358-2365.

21. Kappelman MD, Long MD, Martin C, et al. Evaluation of the patientreported outcomes measurement information system in a large cohort of patients with inflammatory bowel diseases. Clin Gastroenterol Hepatol. 2014;12(8):1315-1323.e2.

22. Larsson K, Lööf L, Rönnblom A, Nordin K. Quality of life for patients with exacerbation in inflammatory bowel disease and how they cope with disease activity. J Psychosom Res. 2008;64(2):139-148.

23. Lix LM, Graff LA, Walker JR, et al. Longitudinal study of quality of life and psychological functioning for active, fluctuating, and inactive disease patterns in inflammatory bowel disease. Inflamm Bowel Dis. 2008; 14(11):1575-1584.

24. Reigada LC, Hoogendoorn CJ, Walsh LC, et al. Anxiety symptoms and disease severity in children and adolescents with Crohn disease. J Pediatr Gastroenterol Nutr. 2015;60(1):30-35.

25. Simrén M, Svedlund J, Posserud I, Björnsson ES, Abrahamsson H. Health-related quality of life in patients attending a gastroenterology outpatient clinic: functional disorders versus organic diseases. Clin Gastroenterol Hepatol. 2006;4(2):187-195.

26. Knowles SR, Wilson J, Wilkinson A, et al. Psychological well-being and quality of life in Crohn's disease patients with an ostomy: a preliminary investigation. J Wound Ostomy Continence Nurs. 2013;40(6):623-629.

27. Ananthakrishnan AN, Gainer VS, Cai T, et al. Similar risk of depression and anxiety following surgery or hospitalization for Crohn's disease and ulcerative colitis. Am J Gastroenterol. 2013;108(4):594-601.

28. Kiebles JL, Doerfler B, Keefer L. Preliminary evidence supporting a framework of psychological adjustment to inflammatory bowel disease. Inflamm Bowel Dis. 2010;16(10):1685-1695.

29. Keegan D, Byrne K, Cullen G, Doherty GA, Dooley B, Mulcahy HE The Stressometer: a simple, valid, and responsive measure of pychological stress in inflammatory bowel disease patients. J Crohns Colitis. 2015;9(10):881-885.

30. Leventhal H, Meyer D, Nerenz D. The common sense model of illness danger. In: Petrie K, Weinman J, editors. Perceptions of Health and Illness. Amsterdam: OPA; 1980:19-46.

31. Hagger MS, Orbell S. A meta-analytic review of the common-sense model of illness representations. Psychol Health. 2003;18(2):141-184.

32. McCombie AM, Mulder RT, Gearry RB. Coping strategies and psychological outcomes of patients with inflammatory bowel disease in the first 6 months after diagnosis. Inflamm Bowel Dis. 2015;21(10): 2272-2280.

33. Heijmans MJ. Coping and adaptive outcome in chronic fatigue syndrome: importance of illness cognitions. J Psychosom Res. 1998;45(1): 39-51.

34. Fortune DG, Richards HL, Griffiths CE, Main CJ. Psychological stress, distress and disability in patients with psoriasis: consensus and variation in the contribution of illness perceptions, coping and alexithymia. $\mathrm{BrJ}$ Clin Psychol. 2002;41(Pt 2):157-174.

35. Scharloo M, Kaptein AA, Weinman J, et al. Illness perceptions, coping and functioning in patients with rheumatoid arthritis, chronic obstructive pulmonary disease and psoriasis. J Psychosom Res. 1998;44(5):573-585.
36. Griva K, Myers LB, Newman S. Illness perceptions and self efficacy in adolescents and young adults with insulin dependent diabetes mellitus. Psychol Health. 2000;15(6):733-750.

37. Hampson SE, Glasgow RE, Strycker LA. Beliefs versus feelings: a comparison of personal models and depression for predicting multiple outcomes in diabetes. BrJ Health Psychol. 2000;5(1):27-40.

38. Heijmans $M$. The role of patients' illness representations in coping and functioning with Addison's disease. BrJ Health Psychol. 1999;4(2): $137-149$.

39. Moss-Morris R, Weinman J, Petrie K, Horne R, Cameron L, Buick D. The revised illness perception questionnaire (IPQ-R). Psychol Health. 2002;17(1):1-16.

40. Rees G, Fry A, Cull A. A family history of breast cancer: women's experiences from a theoretical perspective. Soc Sci Med. 2001;52(9): 1433-1440.

41. Dorrian A, Dempster M, Adair P. Adjustment to inflammatory bowel disease: the relative influence of illness perceptions and coping. Inflamm Bowel Dis. 2009;15(1):47-55.

42. Knowles SR, Wilson JL, Connell WR, Kamm MA. Preliminary examination of the relations between disease activity, illness perceptions, coping strategies, and psychological morbidity in Crohn's disease guided by the common sense model of illness. Inflamm Bowel Dis. 2011; 17(12):2551-2557.

43. Knowles SR, Cook SI, Tribbick D. Relationship between health status, illness perceptions, coping strategies and psychological morbidity: a preliminary study with IBD stoma patients. J Crohns Colitis. 2013;7(10): e471-e478.

44. Best WR, Becktel JM, Singleton JW, Kern F Jr. Development of a Crohn's disease activity index. National cooperative Crohn's disease study. Gastroenterology. 1976;70(3):439-444.

45. Broadbent E, Petrie KJ, Main J, Weinman J. The brief illness perception questionnaire. J Psychosom Res. 2006;60(6):631-637.

46. Carver CS. You want to measure coping but your protocol's too long: consider the brief COPE. Int J Behav Med. 1997;4(1):92-100.

47. Levenstein S, Prantera C, Varvo V, et al. Development of the perceived stress questionnaire: a new tool for psychosomatic research. J Psychosom Res. 1993;37(1):19-32.

48. Zigmond AS, Snaith RP. The hospital anxiety and depression scale. Acta Psychiatr Scand. 1983;67(6):361-370.

49. Guyatt G, Mitchell A, Irvine EJ, et al. A new measure of health status for clinical trials in inflammatory bowel disease. Gastroenterology. 1989; 96(3):804-810.

50. Gibson PR, Vaizey C, Black CM, et al. Relationship between disease severity and quality of life and assessment of health care utilization and cost for ulcerative colitis in Australia: a cross-sectional, observational study. J Crohns Colitis. 2014;8(7):598-606.

51. Nedelciuc O, Pintilie I, Dranga M, Mihai C, Prelipcean CC. Quality of life in patients with ulcerative colitis. Rev Med Chir Soc Med Nat Iasi. 2012;116(3):756-760.

52. Knowles SR, Gass C, Macrae F. Illness perceptions in IBD influence psychological status, sexual health and satisfaction, body image and relational functioning: a preliminary exploration using structural equation modeling. J Crohns Colitis. 2013;7(9):e344-e350.

53. McCombie A, Swaminathan A, Mulder R, Frampton C, Kortlever T, Gearry R. The IBD-cope: a new instrument for measuring coping in inflammatory bowel disease patients. J Crohns Colitis. 2016;10(6): 678-686.

54. Iglesias-Rey M, Barreiro-de Acosta M, Caamaño-Isorna F, et al. Psychological factors are associated with changes in the health-related quality of life in inflammatory bowel disease. Inflamm Bowel Dis. 2014;20(1): 92-102.

55. Tabibian A, Tabibian JH, Beckman LJ, Raffals LL, Papadakis KA, Kane SV. Predictors of health-related quality of life and adherence in Crohn's disease and ulcerative colitis: implications for clinical management. Dig Dis Sci. 2015;60(5):1366-1374.

56. Moser G. Should we incorporate psychological care into the management of IBD? Nat Clin Pract Gastroenterol Hepatol. 2006;3(8):416-417. 
57. Graff LA, Walker JR, Bernstein CN. It's not just about the gut: managing depression and anxiety in inflammatory bowel disease. Pract Gastroenterol. 2010;34(7):11-25.

58. Daghaghzadeh H, Naji F, Afshar H, et al. Efficacy of duloxetine add on in treatment of inflammatory bowel disease patients: a double-blind controlled study. J Res Med Sci. 2015;20(6):595-601.

59. Alarhayem A, Achebe E, Logue AJ. Psychosocial support of the inflammatory bowel disease patient. Surg Clin North Am. 2015;95(6):1281-1293, vii-viii.

60. McCombie A, Gearry R, Andrews J, Mulder R, Mikocka-Walus A. Does computerized cognitive behavioral therapy help people with inflammatory bowel disease? A randomized controlled trial. Inflamm Bowel Dis. 2016;22(1):171-181.

61. Mikocka-Walus A, Bampton P, Hetzel D, Hughes P, Esterman A, Andrews JM. Cognitive-behavioural therapy has no effect on disease activity but improves quality of life in subgroups of patients with inflammatory bowel disease: a pilot randomised controlled trial. BMC Gastroenterol. 2015;15:54.

62. Schoultz M, Atherton I, Watson A. Mindfulness-based cognitive therapy for inflammatory bowel disease patients: findings from an exploratory pilot randomised controlled trial. Trials. 2015;16:379.

63. Tanaka M, Kawakami A, Iwao Y, et al. Descriptive information about Crohn disease: content analysis of patient education brochures. Gastroenterol Nurs. 2010;33(6):432-439.
64. Mofredj A, Alaya S, Tassaioust K, Bahloul H, Mrabet A. Music therapy, a review of the potential therapeutic benefits for the critically ill. J Crit Care. 2016;35:195-199.

65. Sharma P, Poojary G, Dwivedi SN, Deepak KK. Effect of yoga-based intervention in patients with inflammatory bowel disease. Int $J$ Yoga Therap. 2015;25(1):101-112.

66. Denis MA, Reenaers C, Fontaine F, Belaïche J, Louis E . Assessment of endoscopic activity index and biological inflammatory markers in clinically active Crohn's disease with normal C-reactive protein serum level. Inflamm Bowel Dis. 2007;13(9):1100-1105.

67. Durko L, Stasikowska-Kanicka OA, Wagrowska-Danilewicz M, Danilewicz M, Małecka-Panas EI. An analysis of the correlation of clinical, endoscopic and histological classifications in Crohn's disease. Prz Gastroenterol. 2013;8(6):377-382.

68. Yamamoto T, Shiraki M, Bamba T, Umegae S, Matsumoto K. Faecal calprotectin and lactoferrin as markers for monitoring disease activity and predicting clinical recurrence in patients with Crohn's disease after ileocolonic resection: a prospective pilot study. United European Gastroenterol J. 2013;1(5):368-374.
Patient Preference and Adherence

\section{Publish your work in this journal}

Patient Preference and Adherence is an international, peer-reviewed, open access journal that focuses on the growing importance of patient preference and adherence throughout the therapeutic continuum. Patient satisfaction, acceptability, quality of life, compliance, persistence and their role in developing new therapeutic modalities and compounds to optimize

\section{Dovepress}

clinical outcomes for existing disease states are major areas of interest for the journal. This journal has been accepted for indexing on PubMed Central. The manuscript management system is completely online and includes a very quick and fair peer-review system, which is all easy to use. Visit http://www. dovepress.com/testimonials.php to read real quotes from published authors. 